\title{
Subsurface Water Distribution from Drip Irrigation Described by Moment Analyses
}

\author{
N. Lazarovitch,* A. W. Warrick, A. Furman, and J. Šimůnek
}

\begin{abstract}
Moment analysis techniques are used to describe spatial and temporal subsurface wetting patterns resulting from drip emitters. The water added is considered a "plume" with the zeroth moment representing the total volume of water applied. The first moments lead to the location of the center of the plume, and the second moments relate to the amount of spreading about the mean position. We tested this approach with numerically generated data for infiltration from surface and buried line and point sources in three contrasting soils. Ellipses (in two dimensions) or ellipsoids (in three dimensions) can be depicted about the center of the plume. Any fraction of water added can be related to a "probability" curve relating the size of the ellipse (or ellipsoid) that contains that amount of water. Remarkably, the probability curves are identical for all times and all of the contrasting soils. The consistency of the probability relationships can be exploited to pinpoint the extent of subsurface water for any fraction of the volume added. The new method can be immediately applied to the vital question of how many sensors are needed and where to install them to capture the overall water distribution under drip irrigation. For example, better agreement with the "exact" solution occurs with increasing the number of observation points from 6 to 9 and no significant improvement when increasing from 9 to 16 . The method can also be applied to parameter estimation of soil hydraulic properties, which we uniquely reproduced for generated data.
\end{abstract}

$\mathrm{D}$ ESIGNING drip irrigation systems involves selection of an appropriate combination of emitter discharge rate and spacing between emitters for any given set of soil, crop, and climatic conditions, as well as understanding the wetted zone pattern around the emitter (Bresler, 1978; Lubana and Narda, 2001). Water distribution is affected by many factors, including soil hydraulic characteristics, initial conditions, emitter discharge rate, application frequency, root characteristics, evaporation, and transpiration. A traditional way to visualize spatial and temporal soil water distributions includes determination of the water content at points around the emitter and drawing contours between these points. Practical information includes estimation of the position and shape of the wetted volume (Dasberg and Or, 1999). Previous investigators have used descriptions of the extent of wetting, including the surface wetted diameter, wetted depth, and wetted volume (Ben-Asher et al.,

N. Lazarovitch, Wyler Dep. of Dryland Agriculture, Jacob Blaustein Inst. for Desert Research, Ben-Gurion Univ. of the Negev, BeerSheva 84990, Israel; A.W. Warrick, Soil Water and Environmental Science, Univ. of Arizona, Tucson, AZ 85721; A. Furman, Soil, Water and Environmental Sciences, Agricultural Research Organization, Volcani Center, Bet Dagan, 50250 Israel; J. Šimůnek, Dep. of Environmental Science, Univ. of California, Riverside, CA 92521. Received 31 Mar. 2006. *Corresponding author (lazarovi@bgu.ac.il).

Published in Vadose Zone Journal 6:116-123 (2007).

Original Research

doi:10.2136/vzj2006.0052

(c) Soil Science Society of America

677 S. Segoe Rd., Madison, WI 53711 USA
1986; Schwartzman and Zur, 1986; Angelakis et al., 1993; Chu, 1994; Zur, 1996; Dasberg and Or, 1999; Hammami et al., 2002; Thorburn et al., 2003; Cook et al., 2003). The volume of the wetted soil represents the amount of soil water stored in the root zone. The domain of interest should be consistent with the anticipated depth of the root system, while its width is associated with the spacing between emitters and lines (Zur, 1996).

A comprehensive method of characterizing spatialtemporal distributions is through moment analyses. This approach is widely used to describe solute transport in the vadose zone (e.g., Barry and Sposito, 1990; Toride and Leij, 1996; Srivastava et al., 2002). With respect to water, Yeh et al. (2005) and Ye et al. (2005) calculated the zeroth, first, and second moments of a three-dimensional water content plume and defined an ellipsoid that described the average shape and orientation of the plume for each observation period. This led to snapshots of the observed water content plume under transient flow conditions, which was used to derive a three-dimensional effective hydraulic conductivity tensor.

The objective of this study was to implement moment analyses to describe subsurface water distribution resulting from drip irrigation and to test this approach with numerically generated data. This includes infiltration for both line and point sources in contrasting soils. The considerable advantages gained by the moment analyses over alternatives is detailed.

\section{THEORY}

The three-dimensional spatial moments for a water plume, $M_{i j k}$, are defined as (Yeh et al., 2005)

$$
M_{i j k}(t)=\int_{-\infty}^{\infty} \int_{-\infty}^{\infty} \int_{-\infty}^{\infty} \theta_{\text {diff }}(x, y, z, t) x^{i} y^{j} z^{k} \mathrm{~d} x \mathrm{~d} y \mathrm{~d} z
$$

with $\theta_{\text {diff }}(x, y, z, t)=\theta(x, y, z, t)-\theta_{\text {init }}(x, y, z, t)$, where $\theta(x, y, z, t)$ is the water content at a given time $t$ at a location $x, y, z$ and $\theta_{\text {init }}(x, y, z, t)$ is the initial water content, typically small. (Later we will include an example where the initial water content is high enough that redistribution of the antecedent water should be taken into account). The zeroth, first, and second spatial moments correspond to $i+j+k=0,1$, or 2 , respectively. The zeroth moment, $M_{000}$ is the total volume of water applied to the domain. The first moments, $M_{100}, M_{010}$, and $M_{001}$ lead to the location of the center of the plume, and the second moments, $M_{200}, M_{020}$, and $M_{002}$ relate to the amount of spreading about its mean position. The center of mass is at $x_{\mathrm{C}}, y_{\mathrm{C}}, z_{\mathrm{C}}$, given by

$$
x_{\mathrm{C}}=\frac{M_{100}}{M_{000}} \quad y_{\mathrm{C}}=\frac{M_{010}}{M_{000}} \quad z_{\mathrm{C}}=\frac{M_{001}}{M_{000}}
$$


The spread of the plume about its center is described by the spatial variance in the $x, y$, and $z$ directions $\left(\sigma_{x}^{2}\right.$, $\sigma_{y}^{2}$, and $\sigma_{z}^{2}$ ):

$\sigma_{x}^{2}=\frac{M_{200}}{M_{000}}-x_{\mathrm{C}}^{2} \quad \sigma_{y}^{2}=\frac{M_{020}}{M_{000}}-y_{\mathrm{C}}^{2} \quad \sigma_{z}^{2}=\frac{M_{002}}{M_{000}}-z_{\mathrm{C}}^{2}$

For axial symmetry about $r=0$, where $r$ is the radial coordinate $\left(r^{2}=x^{2}+y^{2}\right)$, Eq. [1] can be rewritten by integrating over $r$ and $z$ for the relevant moments. Also by symmetry, $M_{200}$ is equal to $M_{020}$. Thus, $M_{200}$ and $M_{002}$ can be

$$
\begin{aligned}
M_{200}(t) & =\frac{2 \pi}{2} \int_{-\infty}^{\infty} \int_{0}^{\infty} \theta_{\text {diff }}(r, z, t)\left(x^{2}+y^{2}\right) r \mathrm{~d} r \mathrm{~d} z \\
& =\pi \int_{-\infty}^{\infty} \int_{0}^{\infty} \theta_{\text {diff }}(r, z, t) r^{3} \mathrm{~d} r \mathrm{~d} z \\
\mathrm{M}_{002} & =2 \pi \int_{-\infty}^{\infty} \int_{0}^{\infty} \theta_{\text {diff }}(r, z, t) r z^{2} \mathrm{~d} r \mathrm{~d} z
\end{aligned}
$$

\section{MATERIALS AND METHODS}

In the analyses, we used numerical solutions of the Richards equation as implemented in the HYDRUS-2D code (Šimůnek et al., 1999). HYDRUS-2D has been previously used to successfully simulate water flow for drip irrigation systems (Skaggs et al., 2004; Lazarovitch et al., 2005). We demonstrate the moment analyses using four scenarios, including two-dimensional vertical planes with surface line and buried cylindrical cavity sources and three-dimensional axial-symmetrical geometries with surface point and buried spherical cavity sources.

For the surface line and buried cylindrical cavity sources, we used the governing flow equation for two-dimensional isothermal Darcian flow in a variably saturated isotropic rigid porous medium presented by the mixed form of the Richards equation:

$$
\frac{\partial \theta}{\partial t}=\frac{\partial}{\partial x}\left(K \frac{\partial h}{\partial x}\right)+\frac{\partial}{\partial z}\left(K \frac{\partial h}{\partial x}+1\right)
$$

where $\theta$ is the volumetric water content $\left[\mathrm{L}^{3} \mathrm{~L}^{-3}\right], h$ is the soil matric head [L], $K$ is the hydraulic conductivity $\left[\mathrm{L} \mathrm{T}^{-1}\right], x$ and $z$ are spatial coordinates $[\mathrm{L}]$ in transversal and longitudinal directions, respectively ( $z$ positive downward), and $t$ is time [T]

For the surface sources, a specialized version of the code allowed the spatial distribution of the discharge rate at the soil surface to change with time (Gardenas et al., 2005). This was done by switching from a Neumann (flux) to a Dirichlet (head) boundary condition if the surface pressure head required to accommodate the specified flux for a surface node is $>0$. A sufficient number of surface nodes are switched in an iterative way until the entire irrigation flux is accounted for, and the pond width is obtained. Since the infiltration flux into the dry soil is larger for early times, the pond width continuously increases as irrigation proceeds. Other initial and boundary conditions were used to solve numerically Eq. [1]:

$$
\begin{gathered}
h(x, z, t)=h_{\mathrm{i}} \quad t=0 \\
-\frac{\partial h(x, z, t)}{\partial z}=1 \quad \frac{X+x_{0}}{2}<x<\frac{X-x_{0}}{2}, \quad z=0
\end{gathered}
$$

$$
\begin{gathered}
h(x, z, t)=h_{\mathrm{i}} \quad x^{2}+z^{2} \rightarrow \infty \\
\frac{\partial h}{\partial z}=0 \quad z=Z
\end{gathered}
$$

where $x_{0}$ is the pond width [L], $h_{\mathrm{i}}$ is the initial soil pressure head [L], and $Z$ and $X$ are the length and width of the domain, respectively [L].

For the subsurface cavity sources, we used the code from Lazarovitch et al. (2005). This version allows calculation of the source discharge while considering source properties, inlet pressure, and effects of the soil hydraulic properties on the actual discharge.

In the axisymmetrical three-dimensional cases we used

$$
\frac{\partial \theta}{\partial t}=\frac{1}{r} \frac{\partial}{\partial r}\left(r K \frac{\partial h}{\partial r}\right)+\frac{\partial}{\partial z}\left(K \frac{\partial h}{\partial z}\right)-\frac{\partial K}{\partial z}
$$

where $r$ is a radial coordinate [L]. The initial and boundary conditions were analogous to the two-dimensional $(x, z)$ cases

The van Genuchten-Mualem soil hydraulic properties model (Mualem, 1976; van Genuchten, 1980) was selected for the numerical simulations:

$$
\begin{gathered}
S_{\mathrm{e}}=\frac{\theta-\theta_{\mathrm{r}}}{\theta_{\mathrm{s}}-\theta_{\mathrm{r}}}=\left[1+(-\alpha h)^{n}\right]^{-m}, \quad m=1-\frac{1}{n} \\
K=K_{\mathrm{S}} S_{\mathrm{e}}^{0.5}\left[1-\left(1-S_{e}{ }^{1 / m}\right)^{m}\right]^{2}
\end{gathered}
$$

where $S_{\mathrm{e}}$ is the effective fluid saturation [unitless], $h$ is the soil matric head $[\mathrm{L}], \theta_{\mathrm{s}}$ the water contents at saturation $\left[\mathrm{L}^{3} \mathrm{~L}^{-3}\right]$, $\theta_{\mathrm{r}}$ the residual water content $\left[\mathrm{L}^{3} \mathrm{~L}^{-3}\right], K$ the hydraulic conductivity $\left[\mathrm{L} \mathrm{T}^{-1}\right], K_{\mathrm{S}}$ the hydraulic conductivity at saturation $\left[\mathrm{L} \mathrm{T}^{-1}\right.$ ], and $\alpha\left[\mathrm{L}^{-1}\right], n$ [unitless], and $m$ [unitless] are empirical parameters.

The computational domains were selected such that the wetting fronts did not reach the side and the bottom boundaries. The flow domains $(X=1 \mathrm{~m}$ and $Z=1.5 \mathrm{~m}$ in the twodimensional plan and $R=1 \mathrm{~m}$ and $Z=1.5 \mathrm{~m}$ in the threedimensional axial-symmetric plan) were discretized into 1236 nodes with significantly greater detail around the source.

Three homogenous soils with contrasting hydraulic properties were considered. The hydraulic properties of these soils were taken from Carsel and Parrish (1988) and summarized in Table 1. The discharge rate (per unit length) of the surface line source, $Q\left[\mathrm{~L}^{2} \mathrm{~T}^{-1}\right]$, was $0.003 \mathrm{~m}^{2} \mathrm{~h}^{-1}$ and the discharge rate for the point source, $Q\left[\mathrm{~L}^{3} \mathrm{~T}^{-1}\right]$, was $0.001 \mathrm{~m}^{3} \mathrm{~h}^{-1}$. The homogeneous initial effective fluid saturation, $S_{\mathrm{e}}$, was set to 0.01 for all the simulations (except for the cases of where initial conditions were investigated). Simulated application duration was $20 \mathrm{~h}$ for the line sources and $60 \mathrm{~h}$ for the point sources with data saved at 20 evenly spaced times. These application durations lead to an applied volume of $0.06 \mathrm{~m}^{3}$ per $1-\mathrm{m}$ bed in both line and point sources (assuming one emitter per meter), which is the approximate amount of water applied when irrigating for the first time for germination purposes. For the

Table 1. Saturated hydraulic conductivity $\left(K_{S}\right)$, the van GenuchtenMualem fitting parameters $\alpha$ and $n$, and saturated and residual volumetric water contents $\left(\theta_{S}\right.$ and $\theta_{r}$, respectively) for the three representative soils (after Carsel and Parrish, 1988).

\begin{tabular}{lcrlll}
\hline & $K_{\mathrm{S}}$ & $\alpha$ & $n$ & $\theta_{\mathrm{S}}$ & $\theta_{\mathrm{r}}$ \\
\hline & $\mathrm{m} \mathrm{s}^{-1}$ & $\mathrm{~m}^{-1}$ & & \multicolumn{2}{c}{$\mathrm{m}^{3} \mathrm{~m}^{-3}-$} \\
Sand & $\mathbf{8 . 2 5} \times \mathbf{1 0}^{-5}$ & $\mathbf{1 4 . 5}$ & $\mathbf{2 . 6 8}$ & $\mathbf{0 . 4 3}$ & $\mathbf{0 . 0 4 5}$ \\
Sandy loam & $\mathbf{1 . 2 3} \times \mathbf{1 0}^{-5}$ & $\mathbf{7 . 5}$ & $\mathbf{1 . 8 9}$ & $\mathbf{0 . 4 1}$ & $\mathbf{0 . 0 6 5}$ \\
Loam & $\mathbf{2 . 8 9} \times \mathbf{1 0}^{-6}$ & $\mathbf{3 . 6}$ & $\mathbf{1 . 5 6}$ & $\mathbf{0 . 4 3}$ & $\mathbf{0 . 0 7 8}$ \\
\hline
\end{tabular}


cavity sources, the nominal discharge, $Q_{0}$, was $0.003 \mathrm{~m}^{2} \mathrm{~h}^{-1}$ for the two-dimensional cases and $0.001 \mathrm{~m}^{3} \mathrm{~h}^{-1}$ for the threedimensional cases. The radius and depth of the sources were set to 0.01 and $0.15 \mathrm{~m}$, respectively. For expediency, simulations with the subsurface cavity sources were only for the sandy loam soil.

\section{Data Processing}

After completing the simulations, we calculated $M_{i j k}, z_{\mathrm{C}}, \sigma_{x}$, and $\sigma_{z}$ for the two-dimensional plane using Eq. [1] to [3] $\left(x_{\mathrm{C}}\right.$ was equal to zero). For the axially symmetric case, $M_{i j k}, z_{\mathrm{C}}, \sigma_{x}$, and $\sigma_{z}$ were found from Eq. [1] to [5]. A separate program was used to compute the moments directly from the HYDRUS-2D output. For expediency, an equally spaced grid was defined and values of water content in the grid points were assumed to be that of the closest finite element node. The moments were then computed using the gridded values. To check for mass balance, the values of $M_{000}$ in all cases were verified to agree within $0.1 \%$ of the applied water.

After calculating the moments for each case and time, we defined ellipses around the center of mass $\left(0, z_{\mathrm{C}}\right)$ in the twodimensional plane. The semi-axes of the ellipses are analogous to the semi-axes of a binormal probability distribution (e.g., Morrison, 1976, Ch. 3). For the two-dimensional case, we used $k \sigma_{x}$ and $k \sigma_{z}$, where $k$ is the number of standard deviations. Each ellipse was set as

$$
\frac{x^{2}}{k^{2} \sigma_{x}^{2}}+\frac{z^{2}}{k^{2} \sigma_{z}^{2}}=1
$$

In the axially symmetric cases, we defined spheroids (symmetrical about the $z$ axis with semi-axes $\sigma_{x}, \sigma_{y}=\sigma_{x}$ and $\sigma_{z}$ ):

$$
\frac{x^{2}}{k^{2} \sigma_{x}^{2}}+\frac{y^{2}}{k^{2} \sigma_{x}^{2}}+\frac{z^{2}}{k^{2} \sigma_{z}^{2}}=\frac{r^{2}}{k^{2} \sigma_{x}^{2}}+\frac{z^{2}}{k^{2} \sigma_{z}^{2}}=1
$$

The amount of water within an ellipse was also computed using the gridded-water content values with Eq. [1]. This was done using the same program used to generate the moment values. Dividing the mass of added water within any ellipse by $M_{000}$ gives a corresponding fraction of the total water applied. By repeating the calculations for increasing values of $k$, the result is a cumulative probability function, $P$, going from $P=0$ for $k=0$ (none of the added water is included within a point) to $P=1$ as $k$ becomes large (all of the added water is included as an ellipse or spheroid becomes large). Finally, cumulative beta distribution functions were fitted with

$$
P(u)=\frac{(1-u)^{b-1} u^{a-1}}{B(a, b)}
$$

where $\mathrm{B}(a, b)$ is the complete beta function, $a$ and $b$ are parameters, and $u=k / k_{\max }$ (an appropriate value of $k_{\max }=3$ is used below). Results for the probability function and its interpretation will be given below.

For calculating $M_{i j k}$, we assumed that the initial water content, $\theta_{\text {init }}$, was sufficiently small that redistribution of the antecedent water was negligible over the time frames considered. For wetter initial conditions, however, significant redistribution of antecedent water can occur due to gravity drainage. This can lead to negative values for the integrals in Eq. [1]. For this condition, we propose to use the background water content $\left(\theta_{\mathrm{bg}}\right)$ rather than $\theta_{\text {init }}$ for calculating $\theta_{\text {diff }}$ in Eq. [1]. The background water content is time and depth dependent and is defined as the equivalent water content after redistribution starting from the same initial conditions. The calculations are performed for the same domain and initial and boundary conditions but without adding any water.

\section{RESULTS AND DISCUSSION}

For the two-dimensional domain, the center of mass, $z_{\mathrm{C}}$, as a function of time for the three representative soils is presented in Fig. 1A. As time progresses, water advances from the surface source deeper into the profile, well depicted by following values of $z_{\mathrm{C}}$. In the beginning of the irrigation event, $z_{\mathrm{C}}$ advances fast and after several hours the rate of the advance becomes nearly constant. The contrast in texture leads to different retention capacities for the soils. The coarse-textured sand has the lowest retention capacity and the deepest $z_{C}$; the fine-textured loam has the highest retention capacity with shallowest $z_{\mathrm{C}}$.

The semi-axes in $x$ and $z$ directions, $\sigma_{x}$ and $\sigma_{z}$ (as a function of time) are depicted in Fig. 1B and 1C, respectively. While $\sigma_{z}$ exhibits differences between the soils, $\sigma_{x}$ reveals almost the same values for the sandy and the sandy loam soils but slightly higher values for the loamy soil at any given time.

Wetting patterns and the ellipses for one and two standard deviations about $z_{\mathrm{C}}$ are illustrated in Fig. 2 at $20 \mathrm{~h}$. Of course, these ellipses are consistent with Fig. 1. For example, $z_{\mathrm{C}}=-0.39 \mathrm{~m}, \sigma_{x}=0.14 \mathrm{~m}$, and $\sigma_{z}=$ $0.22 \mathrm{~m}$ for the sandy soil with $t=20 \mathrm{~h}$. These ellipses can be shown also for early times using the same procedure. The shape of the ellipses varies with soil texture. While the ellipses in the sandy soil are highly elongated, the ellipses in the sandy loam soil are close to circles.

The center of mass, $z_{\mathrm{C}}$, for surface point sources for the three representative soils is presented in Fig. 3A.
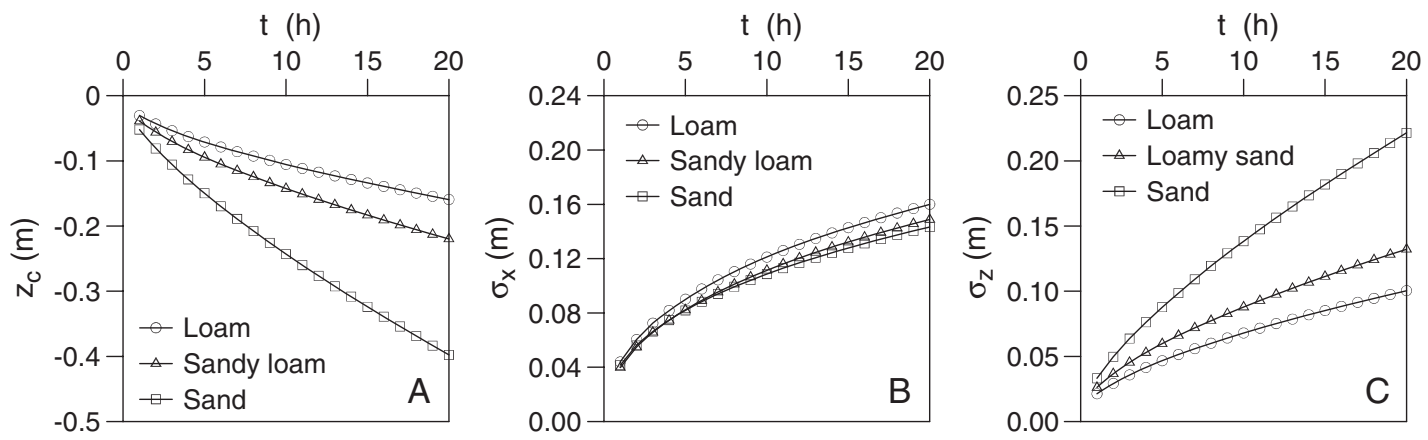

Fig. 1. (A) The vertical center of gravity, $z_{\mathrm{C}}$, for a surface point source in a two-dimensional plane as a function of time, $t$, for three representative soils; (B) spatial variance in the $x$ direction, $\sigma_{x}$, as a function of $t$; and (C) spatial variance in the $z$ direction, $\sigma_{z}$, as a function of $t$. 

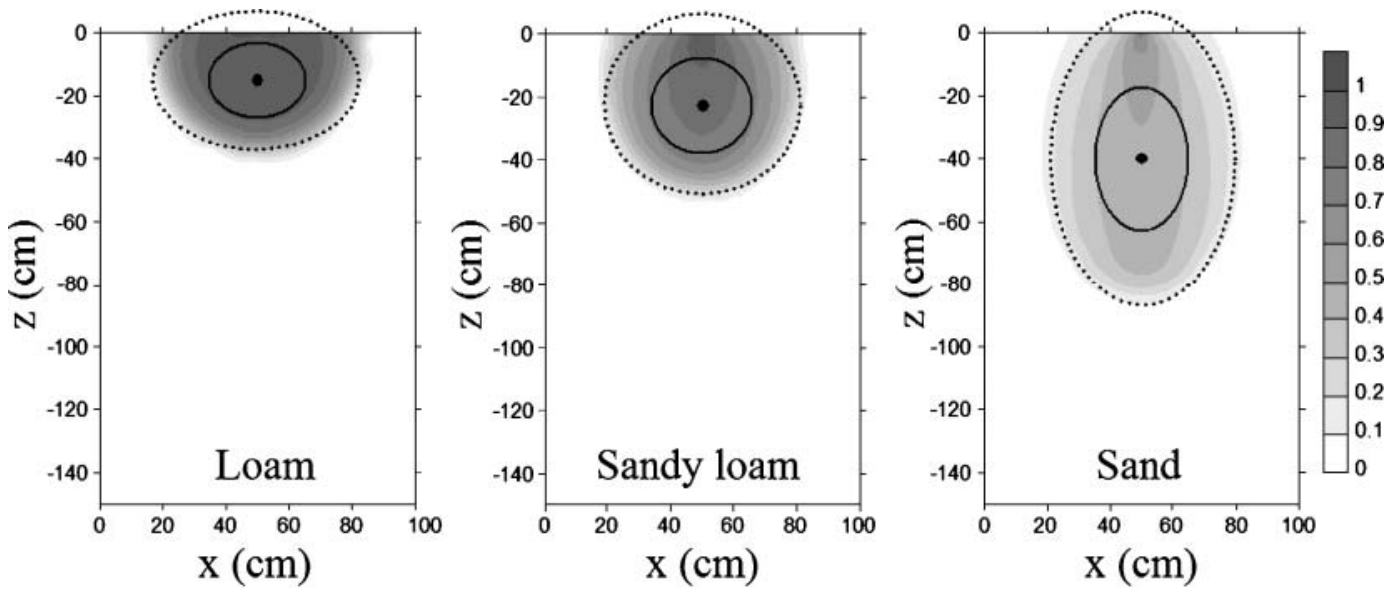

Fig. 2. Wetting patterns and ellipses for one (solid line) and two (dashed line) standard deviations about the vertical center of gravity (small filled circle), for three representative soils under a surface line source.

The semi-axes in $z$ and $x$ directions, $\sigma_{x}$ and $\sigma_{z}$, are presented in Fig. 3B and 3C, respectively. The $z_{\mathrm{C}}$ in the three-dimensional cases shows the same properties as in the two-dimensional cases in that the coarser the texture, the deeper the $z_{\mathrm{C}}$. Also, the horizontal variances are nearly independent of texture, whereas the vertical variance shows differences similar to those for the twodimensional case.

Wetting patterns and the resulting spheroids for the surface point applications are illustrated in Fig. 4 at the end of irrigation $(60 \mathrm{~h})$. Results are shown in the $x-z$ plane, with the resulting ellipses (these are crosssections of the corresponding spheroids) for one and two standard deviations (alternatively, the $x$ axis may be considered as an $r$ axis). As in the previous examples, the finer texture (loam) results in a distribution pattern nearer to the surface, whereas the coarser textured sand has pattern that is deeper in the profile.

For the subsurface cavity sources, distribution patterns and the resulting ellipses were calculated only for the sandy loam. Both the two- and three-dimensional results are shown in Fig. 5 for $40 \mathrm{~h}$.

Cumulative probability as a function of the number of standard deviations for various soils, times, and dimensions of the irrigation event is presented in Fig. 6. Although $z_{\mathrm{C}}, \sigma_{z}$, and $\sigma_{x}$ differ for each soil and time, the fraction of the amount of water within ellipses or spheroids from the total applied water is, for practical pur- poses, the same. Increasing the size of the ellipses (or spheroids) includes a higher fraction of the water in the plume. Essentially, all of the added water resides within the ellipses or spheroids defined by the number of standard deviations $k=3$. The solid curves are fitted using the beta distribution Eq. [21]. The fitted parameters for the two-dimensional case are $a=3.15, b=3.98$, and for the three-dimensional case are $a=4.33$ and $b=3.46$.

The fact that the curve for the three-dimensional case is below the two-dimensional results in Fig. 6 could have been anticipated based on an analogy to general probability distributions. To demonstrate this for a simple case, compare normal, binormal, and trinormal distributions with independent variables. For the normal distribution the probability that a random variable $|X|$ (of mean 0 ) is less than the standard deviation is well known to be 0.683 ; for two standard deviations, the probability is 0.954 (see Table 2). Analogous results for the binormal distribution (assuming independence of variables) are found and represent the probability space within a circle of radius $k \sigma$. The circular space is conveniently approximated by taking a square that has the same area as the circle or taking $|X|$ and $|Y|<0.5 \pi^{0.5} \mathrm{k}$. If the two variables are assumed independent, the individual probabilities are multiplied together, giving the expression in the first column of Table 2 and numerical values of 0.39 and 0.853 for one and two stan-
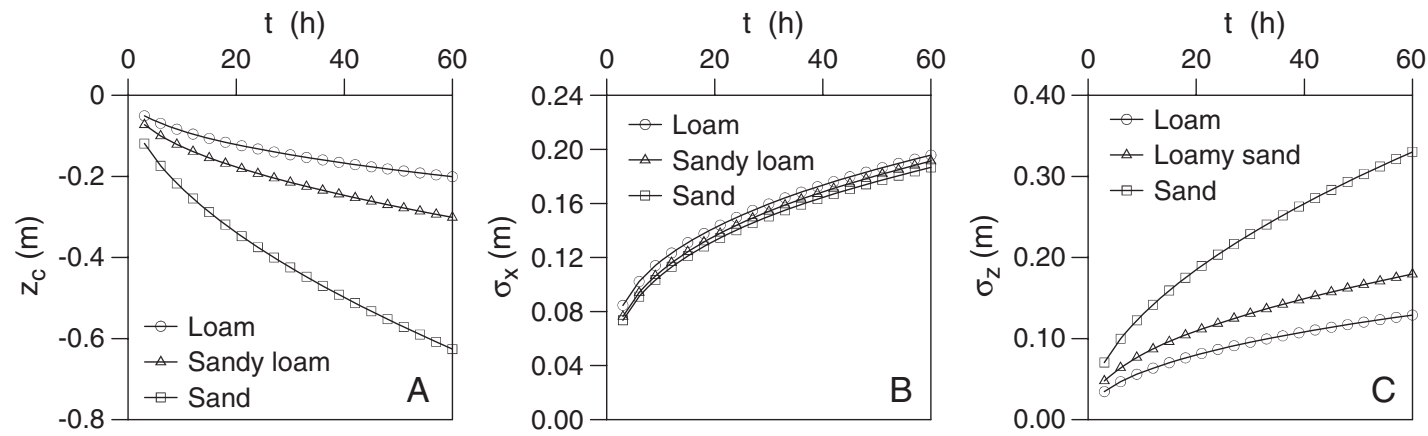

Fig. 3. (A) The vertical center of gravity, $z_{\mathrm{C}}$, for a surface point source in a three-dimensional plane as a function of time, $t$, for three representative soils; (B) spatial variance in the $x$ direction, $\sigma_{x}$, as a function of $t$; and (C) spatial variance in the $z$ direction, $\sigma_{z}$, as a function of $t$. 

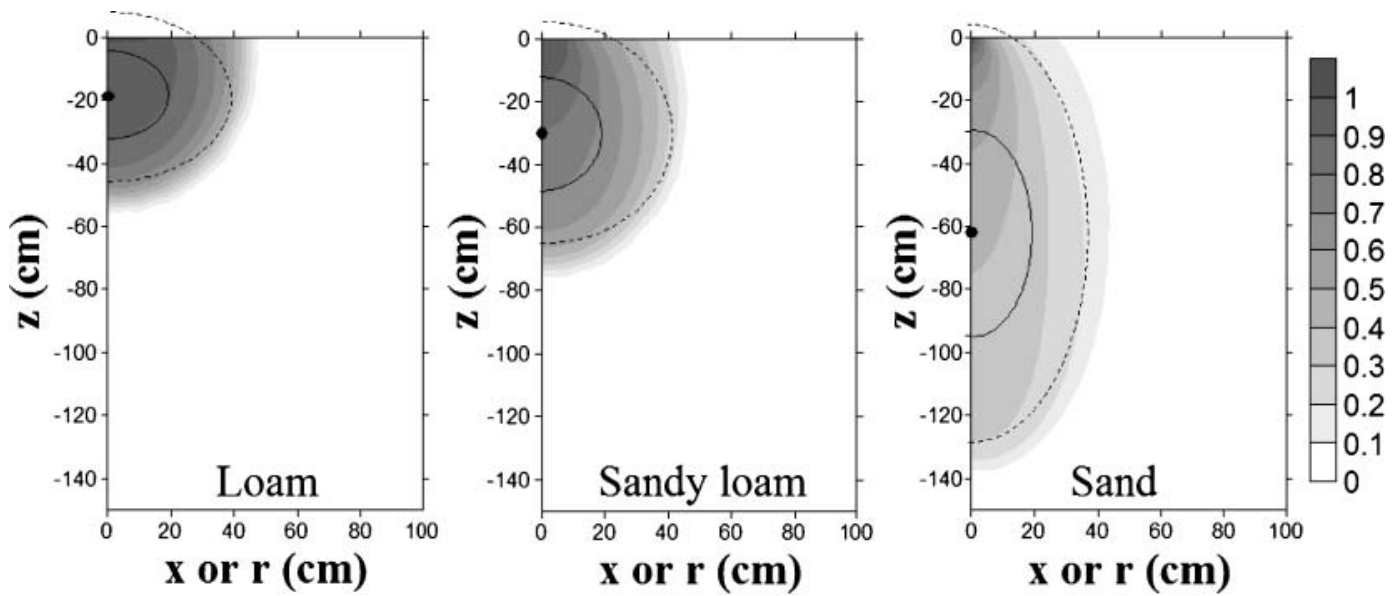

Fig. 4. Cross-sections of the wetting patterns and the spheroids for one (solid line) and two (dashed line) standard deviations about the vertical center of gravity (small filled circle), for three representative soils under a surface point source.

dard deviations. For the trinormal distribution, a similar approximation is made that a sphere of radius $k \sigma$ can be replaced by a cube of the same volume leading to the probability given in the first column of Table 2 with the resulting values of 0.195 and 0.712 . Values from Fig. 6 are shown in parentheses and are slightly smaller for $k=1$ (e.g., 0.303 compared with 0.390 for the twodimensional case) and slightly larger for $k=2(0.892$ compared with 0.853 ). The uniqueness of the probability curves defined by the ellipses was not anticipated. This allows very detailed definitions of the subsurface water using the time distributions of only three parameters: $z_{\mathrm{C}}$, $\sigma_{z}$, and $\sigma_{x}$. In fact, a reasonable approximation may be to use a single curve (with time) for the horizontal variances as evidenced by Fig. 1B and 3B.

We used $\theta_{\mathrm{bg}}$ for calculating $z_{\mathrm{C}}$ for a surface point source with discharge rate of $0.001 \mathrm{~m}^{3} \mathrm{~h}^{-1}$ in a sandy soil having initial saturations, $S_{\mathrm{e}}$, of $0.01,0.05$, and 0.1 . We chose the sandy soil since it yields the highest changes in water contents when the initial water is redistributing in the soil during the irrigation event. The results are shown in Fig. 7. For $S_{\mathrm{e}}=0.01$ the results are consistent with Fig. 3 but for $S_{\mathrm{e}}=0.05$ and $0.1, z_{\mathrm{C}}$ is deeper as expected.
A possible use of the moment analysis method is to address the vital question of how many sensors are needed and where to install them to capture the overall plume behavior in drip irrigation. The traditional method is to place one to three sensors (tensiometers, time domain reflectometry probes, etc.), hoping to capture the entire root zone water dynamics (e.g., Dasberg and Or, 1999). Rarely more than one sensor is placed, and in such cases the water content is simply averaged. We suggest here that by calculating spatial moments and generating ellipses or spheroids as described above, the true distribution of the water as it resides in the subsurface can be captured.

Figure 8 shows spheroids about $z_{\mathrm{C}}$ for 6,9 , and 16 observation points that were distributed evenly in the domain of interest. These spheroids' curves (dashed lines) are comparable to the "exact" spheroids (solid lines) for 50 and $90 \%$ of the water in the domain. The results are for the surface point source into the loamy soil with a discharge rate of $0.0005 \mathrm{~m}^{3} \mathrm{~h}^{-1}$ with $S_{\mathrm{e}}=0.01$ after $40 \mathrm{~h}$. The "exact" curve was calculated using all the nodal information. In the other cases, the moments were calculated by assigning an area (calculated with rectangles and presented in Fig. 8 with dashed lines) to each
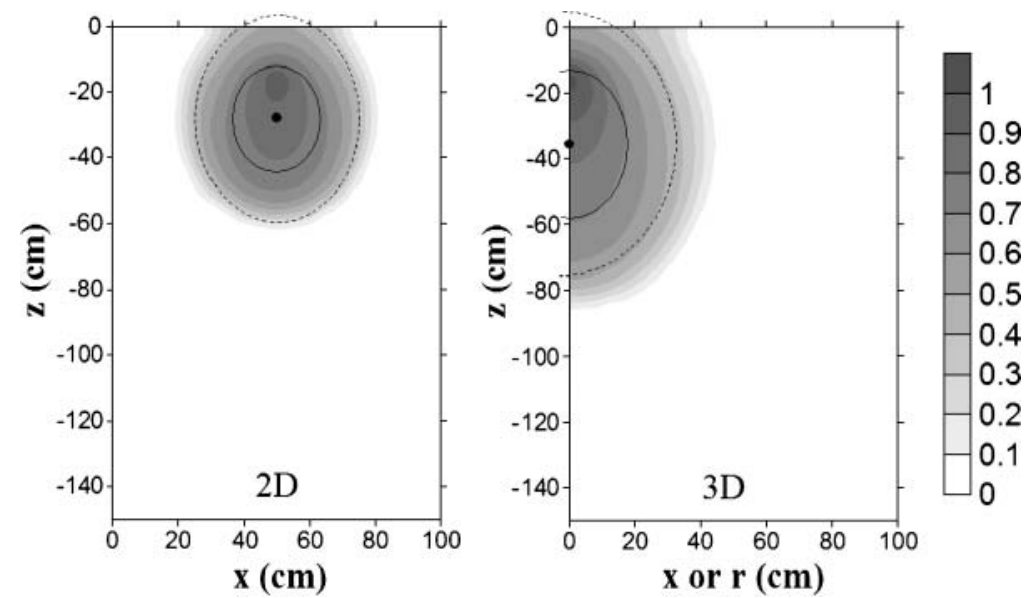

Fig. 5. Wetting patterns and ellipses for one (solid line) and two (dashed line) standard deviations about the vertical center of gravity, $z_{\mathrm{C}}$ (small full circle), for a subsurface cavity source in two dimensions (2D) and the analogous results for a three-dimensional (3D) cavity for a loamy sand soil. 


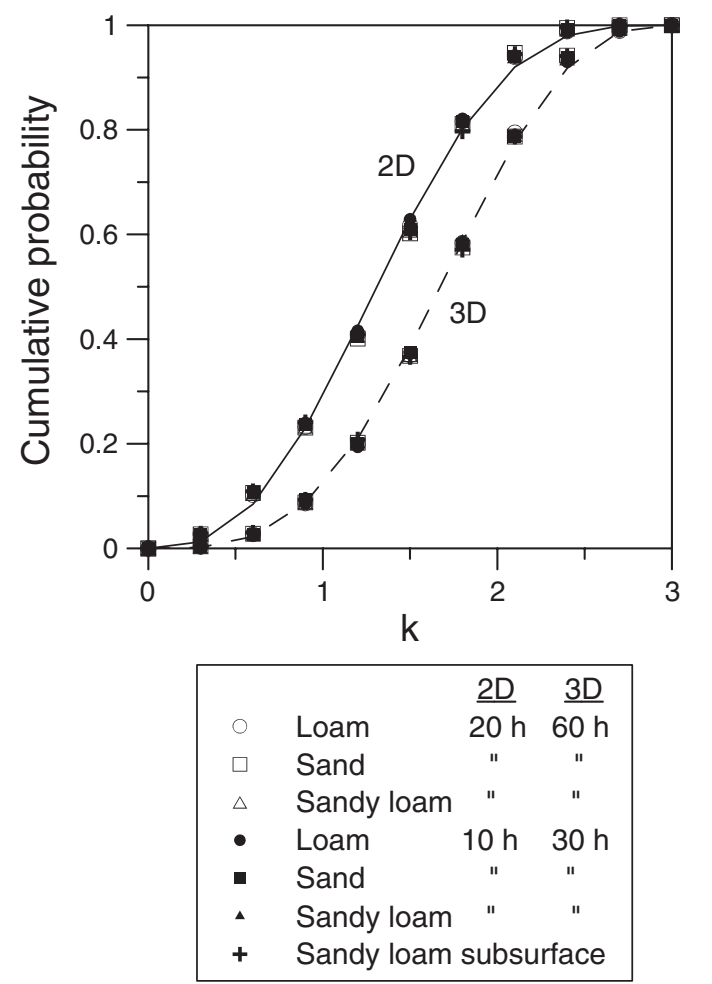

Fig. 6. Cumulative probability as a function of the number of standard deviations, $k$, for various soils, times, source locations, and two (2D) or three dimensions (3D).

observation point (presented as a triangle) and assuming that in this area the water content is constant and equal to the water content of the observation point. Better agreement with the "exact" solution occurs with an increased number of observation points from six to nine, but no significant improvement when increasing from 9 to 16.

The moment analyses can also be applied to parameter estimation of the soil hydraulic properties. Parameter estimation methods have been extensively applied to determine soil hydraulic properties (Russo et al., 1991; Hopmans et al., 2002). Usually the input data for the parameter estimation consist of water content, water potential, or infiltrations. The knowledge from the moment analyses can serve as an alternative input for the parameter estimation. In the next analysis, we tried to estimate $\alpha$ and $n$ of the loamy soil. We assumed that we know the rest of the hydraulic parameters of this soil (i.e., $K_{\mathrm{s}}, \theta_{\mathrm{s}}$, and $\theta_{\mathrm{r}}$ ) and we also know the information about $z_{\mathrm{C}}(t), \sigma_{r}(t)$ and $\sigma_{z}(t)$. We used $64(8 \times 8)$ simulations of surface point sources with $Q=0.001 \mathrm{~m}^{3} \mathrm{~h}^{-1}$ to create a response surface between the simulated data

Table 2. Probability of being within one $(k=1)$ and two $(k=2)$ standard deviations of the mean for normal, binormal, and trinormal normal distributions. The values in parentheses are from Fig. 6.

\begin{tabular}{lll}
\hline Distribution $\dagger$ & \multicolumn{1}{c}{$k=1$} & \multicolumn{1}{c}{$k=2$} \\
\hline Normal $P(|X|<k \sigma)$ & 0.683 & 0.954 \\
Binormal $\left\{P\left[|X|<\left(\pi^{0.5} k \sigma\right) / 2\right]\right\}^{2}$ & $0.390(0.303)$ & $0.853(0.892)$ \\
Trinormal $\left\{P\left[|X|<(\pi / 6)^{1 / 3} k \sigma\right]\right\}^{3}$ & $0.195(0.118)$ & $0.712(0.724)$ \\
\hline
\end{tabular}

$\dagger \boldsymbol{P}=$ probability, $X=$ random variable, $\sigma=$ spatial variance.

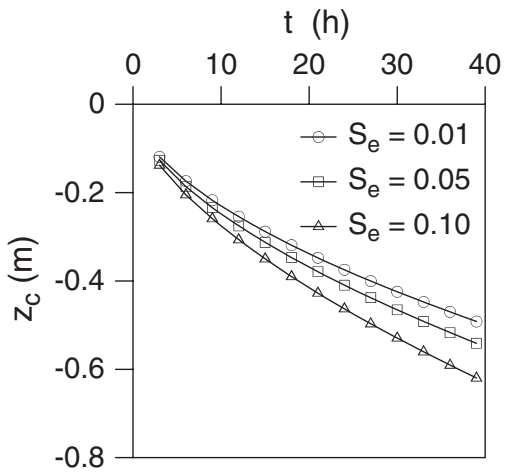

Fig. 7. The vertical center of gravity, $z_{C}$, for the surface point source as a function of time, $t$, for the sandy soil with three initial saturations $\left(S_{\mathrm{e}}\right)$.

and the unknown soil. The sum of squared errors $(\Phi)$ was calculated with

$$
\Phi=\sum_{i=1}^{b}\left(z_{\mathrm{Ci}}-z_{\mathrm{Ci}} *\right)^{2}+\left(\sigma_{z \mathrm{i}}-\sigma_{z \mathrm{i}} *\right)^{2}+\left(\sigma_{r \mathrm{i}}-\sigma_{r \mathrm{i}} *\right)^{2}
$$

where $b$ is the number of print times, the asterisks denote the "measured point," and the subscript i denotes and index for a particular calculated point. Figure 9 shows $\Phi$ as a function of $\alpha$ and $n$. The minima of the error is exactly with $\alpha=3.6 \mathrm{~m}$ and $n=1.56$. This unique reproduction of $\alpha$ and $n$ for the loamy soil suggests the use of the moment analyses with inverse estimation of soil hydraulic properties.

\section{SUMMARY AND CONCLUSIONS}

Spatial moments describing subsurface water distribution from drip sources are presented for numerically generated data. These analyses allow a straightforward, physically meaningful description of the general pattern of water distribution around surface and subsurface sources. As opposed to traditional methods, which require detailed information of the water content distribution, moment analyses can accurately describe the water content distribution in a statistical manner with just three numbers $\left(z_{\mathrm{C}}, \sigma_{z}\right.$, and $\left.\sigma_{x}\right)$. Remarkably, the probability function relating fraction of water applied to an appropriate encapsulating ellipse (or spheroid) is not soil or time dependent. Thus, once $z_{\mathrm{C}}, \sigma_{z}$, and $\sigma_{x}$ are known, the overall boundaries and shape of the wetted volume can be approximated with a high accuracy. For any specified fraction of the volume of water added, an ellipse (or spheroids) is easily defined within which a corresponding proportion of the added water resides.

In implementing moment analyses to subsurface water distribution, the emphasis has been primarily on definitions and evaluation of the moments. Unlike other examples of moment analysis in vadose zone hydrology, the results are unique in that here the total water in the subsurface plume is increasing due to additional water being added to the system. Numerous questions arise as to how to address technical problems and how to best exploit the techniques. Although considerable informa- 

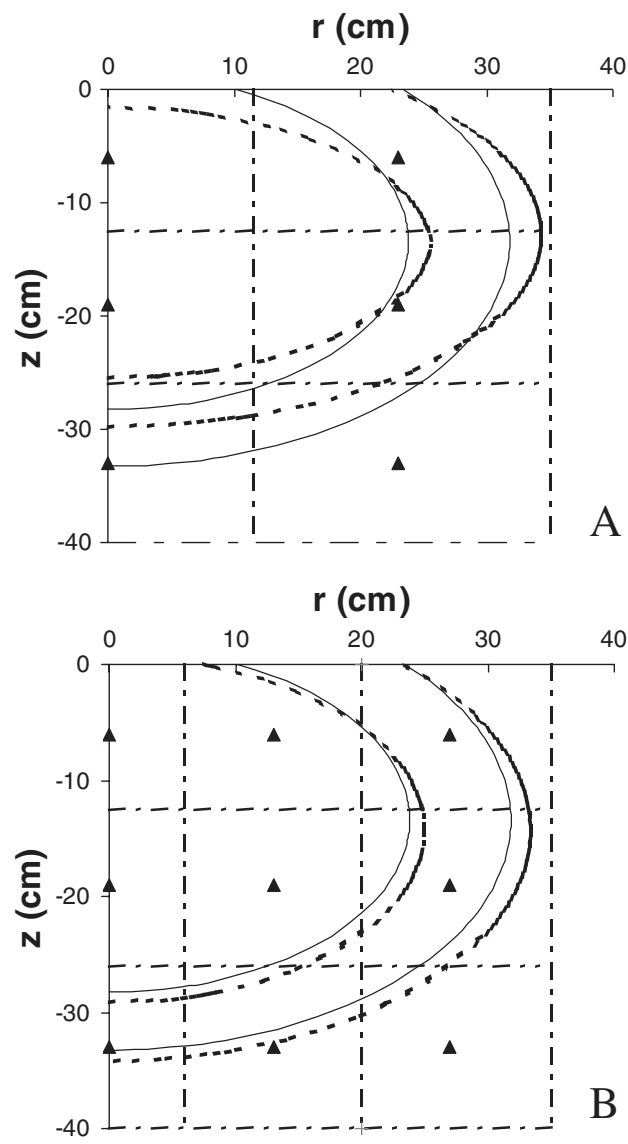

$r(\mathrm{~cm})$

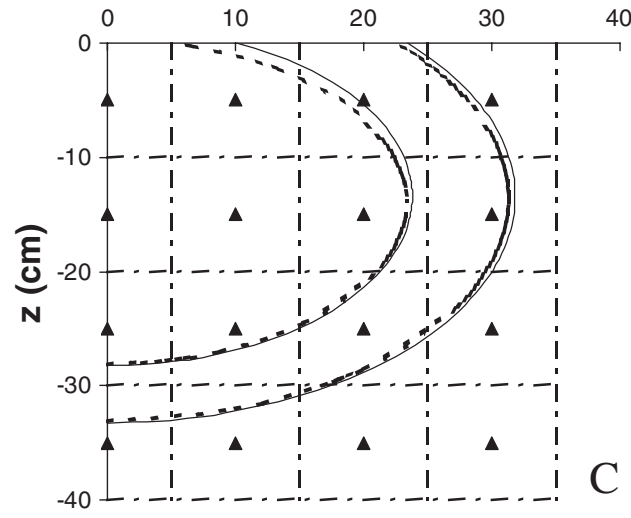

Fig. 8. Cross-sections of the spheroids (dashed lines) about the vertical center of gravity for (A) 6, (B) 9, and (C) 16 observation points (triangles) compared with the "exact" results (solid lines) under a surface point source. The associated Thiessen polygons are showed as dashed lines.

tion follows from knowing $z_{\mathrm{C}}, \sigma_{z}$, and $\sigma_{x}$, their values change with time; hence, more direct methods for evaluation would be extremely useful. The two applications (sensor evaluation and inverse parameter estimation) deserve further attention in the future, which is beyond the scope of this paper. Other geometries and processes, such as flow from channels and boreholes, as well as for water redistribution and water uptake by plants, also appear to be fruitful topics to receive attention in the future.

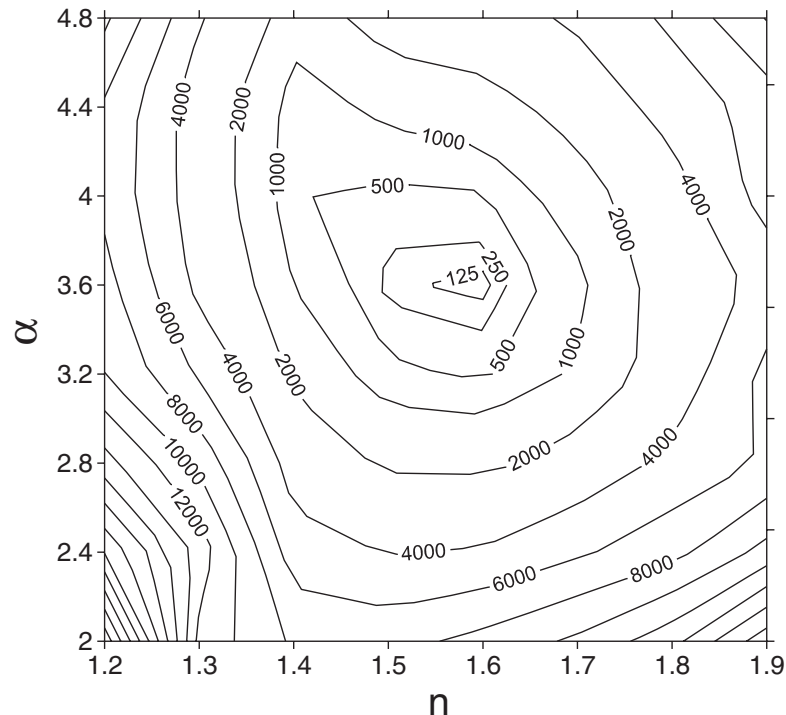

Fig. 9. The sum of squared errors, $\Phi$, as a function of the fitting parameters $n$ and $\alpha\left(\mathrm{m}^{-1}\right)$ for the hypothetical case of the loamy soil.

\section{ACKNOWLEDGMENTS}

This work was supported by The United States-Israel Binational Agricultural Research and Development fund (BARD), Project Grant Agreement no. US-3662-05R and Western Research Project W-1188. Grateful acknowledgments are made to Don Myers and Jim Yeh for helpful suggestions with respect to the moment definitions.

\section{REFERENCES}

Angelakis, A.N., D.E. Rolston, T.N. Kadir, and V.N. Scott. 1993. Soil-water distribution under trickle source. J. Irrig. Drain. Eng. 119:484-500.

Barry, D.A., and G. Sposito. 1990. Three-dimensional statistical moment analysis of the Stanford/Waterloo Borden tracer data. Water Resour. Res. 26:1735-1747.

Ben-Asher, J., C. Charach, and A. Zemel. 1986. Infiltration and water extraction from trickle irrigation source. The effective hemisphere model. Soil Sci. Soc. Am. J. 50:882-887.

Bresler, E. 1978. Analysis of trickle irrigation with application to design problems. Irrig. Sci. 1:3-17.

Carsel, R.F., and R.S. Parrish. 1988. Developing joint probability distributions of soil water retention characteristics. Water Resour. Res. 24:755-769.

Chu, S.T. 1994. Green-Ampt analysis of wetting patterns for surface emitters. J. Irrig. Drain. Eng. 120:414-421.

Cook, F.J., P.J. Thorburn, P. Fitch, and K.L. Bristow. 2003. WetUp: A software tool to display approximate wetting patterns from drippers. Irrig. Sci. 22:129-134.

Dasberg, S., and D. Or. 1999. Drip irrigation. Springer Verlag, Berlin. Gardenas, A.I., J.W. Hopmans, B.R. Hanson, and J. Šmůnek. 2005. Two-dimensional modeling of nitrate leaching for various fertigation scenarios under micro-irrigation. Agric. Water Manage. 74: 219-242.

Hammami, M., H. Daghari, J. Balti, and M. Maalej. 2002. Approach for predicting the wetting front depth beneath a surface point source: Theory and numerical aspect. Irrig. Drain. 51:347-360.

Hopmans, J.W., J. Šmůnek, N. Romano, and W. Durner. 2002. Simultaneous determination of water transmission and retention properties. Inverse methods. p. 963-1008. In J.H. Dane and G.C. Topp (ed.) Methods of soil analysis. Part 4. SSSA Book Ser. 5. SSSA, Madison, WI.

Lazarovitch, N., J. Šimůnek, and U. Shani. 2005. System-dependent boundary condition for water flow from subsurface source. Soil Sci. Soc. Am. J. 69:46-50.

Lubana, P.P.S., and N.K. Narda. 2001. Soil and water modelling. Soil water dynamics under trickle emitters-A review. J. Agric. Eng. Res. 78:217-232. 
Morrison, D.F. 1976. Multivariate statistical methods. McGraw-Hill, New York.

Mualem, Y. 1976. A new model for predicting the hydraulic conductivity of unsaturated porous media. Water Resour. Res. 12: 513-522.

Russo, D., E. Bresler, U. Shani, and J.C. Parker. 1991. Analyses of infiltration events in relation to determining soil hydraulic properties by inverse problem methodology. Water Resour. Res. 27: 1361-1373.

Schwartzman, M., and B. Zur. 1986. Emitter spacing and geometry of wetted soil volume. J. Irrig. Drain. Eng. 112:242-253.

Šimůnek, J., M. Sejna, and M.Th. van Genuchten. 1999. The HYDRUS-2D software package for simulating two-dimensional movement of water, heat, and multiple solutes in variably saturated media. Version 2.0. Rep. IGWMC-TPS-53. Int. Ground Water Model. Ctr., Golden, CO.

Skaggs, T.H., T.J. Trout, J. Šimůnek, and P.J. Shouse. 2004. Comparison of HYDRUS-2D simulations of drip irrigation with experimental observations. J. Irrig. Drain. Eng. 130:304-310.

Srivastava, R., P.K. Sharma, and M.L. Brusseau. 2002. Spatial mo- ments for reactive transport in heterogeneous porous media. J. Hydrol. Eng. 7:336-341.

Thorburn, P.J., F.J. Cook, and K.L. Bristow. 2003. Soil-dependent wetting from trickle emitters: Implications for system design and management. Irrig. Sci. 22:121-127.

Toride, N., and F.J. Leij. 1996. Convective-dispersive stream tube model for field-scale solute transport: I. Moment analysis. Soil Sci. Soc. Am. J. 60:342-352.

van Genuchten, M.Th. 1980. A closed-form equation for predicting the hydraulic conductivity of unsaturated soils. Soil Sci. Soc. Am. J. 44:892-898

Ye, M., R. Khaleel, and T.C.J. Yeh. 2005. Stochastic analysis of moisture plume dynamics of a field injection experiment. Water Resour. Res. 41(3):W03013, doi:10.1029/2004WR003735.

Yeh, T.C.J., M. Ye, and R. Khaleel. 2005. Estimation of effective unsaturated hydraulic conductivity tensor using spatial moments of observed moisture plume. Water Resour. Res. 41(3):W03014, doi:10.1029/2004WR003736.

Zur, B. 1996. Wetted soil volume as a design objective in trickle irrigation. Irrig. Sci. 16:101-105. 